OPEN ACCESS

Edited by: Antonio Ruggiero, Catholic University of the

Sacred Heart, Italy

Reviewed by: Iwaho Kikuchi,

Juntendo University, Japan Enes Taylan,

Mount Sinai Hospital, United States

${ }^{*}$ Correspondence: Kenny A. Rodriguez-Wallberg kenny.rodriguez-wallberg@ki.se

Specialty section:

This article was submitted to Pediatric Oncology, a section of the journal Frontiers in Oncology

Received: 18 March 2021 Accepted: 02 September 2021 Published: 24 September 2021

Citation:

Hao X, Anastácio A, Viñals-Ribé L, Santamaria Lacuesta $A$, Diakaki C, Alonso de Mena S, Liu K and Rodriguez-Wallberg KA (2021)

Follicle Rescue From Prepubertal Ovaries After Recent Treatment With Cyclophosphamide-

An Experimental Culture System

Using Mice to Achieve Mature Oocytes for Fertility Preservation.

Front. Oncol. 11:682470. doi: 10.3389/fonc.2021.682470

\section{Follicle Rescue From Prepubertal Ovaries After Recent Treatment With Cyclophosphamide-An Experimental Culture System Using Mice to Achieve Mature Oocytes for Fertility Preservation}

\author{
Xia Hao ${ }^{1,2}$, Amandine Anastácio ${ }^{1,2}$, Laia Viñals-Ribé ${ }^{1,2}$, Ana Santamaria Lacuesta ${ }^{1,2}$, \\ Christina Diakaki ${ }^{1,2}$, Sara Alonso de Mena ${ }^{1,2}$, Kui Liu ${ }^{3,4}$ and Kenny A. Rodriguez-Wallberg ${ }^{1,2,5 *}$ \\ 1 Department of Oncology and Pathology, Karolinska Institutet, Stockholm, Sweden, ${ }^{2}$ Laboratory of Translational Fertility \\ Preservation, BioClinicum, Stockholm, Sweden, ${ }^{3}$ Shenzhen Key Laboratory of Fertility Regulation, Center of Assisted \\ Reproduction and Embryology, The University of Hong Kong-Shenzhen Hospital, Shenzhen, China, ${ }^{4}$ Department of \\ Obstetrics and Gynecology, Li Ka Shing Faculty of Medicine, The University of Hong Kong, Hong Kong, Hong Kong, \\ SAR China, ${ }^{5}$ Department of Reproductive Medicine, Division of Gynecology and Reproduction, Karolinska University \\ Hospital, Stockholm, Sweden
}

Ovarian tissue cryopreservation is the only feasible method for fertility preservation in prepubertal girls that will undergo gonadotoxic chemotherapy. To date, the only clinical use of cryopreserved tissue is by a later tissue retransplantation to the patient. Clinical challenges in fertility preservation of very young patients with cancer include time constraints that do not allow to retrieve the tissue for cryopreservation before starting chemotherapy and the preclusion of future ovarian tissue transplantation due to the risk of reintroduction of malignant cells in patients with systemic diseases. To overcome these two challenges, we investigated using an experimental model the feasibility of retrieving secondary follicles from ovaries of prepubertal mice after cyclophosphamide (CPA) treatment in increasing doses of 50,75 , and $100 \mathrm{mg} / \mathrm{kg}$. The follicles were thereafter cultured and matured in vitro. The main outcomes included the efficiency of the method in terms of obtained matured oocytes and the safety of these potentially fertility preservative procedures in terms of analyses of oocyte competence regarding normality of the spindle and chromosome configurations. Our findings demonstrated that it was feasible to isolate and culture secondary follicles and to obtain mature oocytes from prepubertal mice ovaries recently treated with CPA. The efficiency of this method was highly demonstrated in the $100 \mathrm{mg} / \mathrm{kg}$ CPA group, with near 90\% follicle survival rate after 12 days' culture, similarly to control. Around $80 \%$ of the follicles met the criteria to put into maturation, and more than $40 \%$ of them achieved metaphase II, with normal spindle and chromosome configurations observed. Suboptimal results were obtained in the 50 and $75 \mathrm{mg} / \mathrm{kg} \mathrm{CPA}$ 
groups. These paradoxical findings towards CPA dose might probably reflect a more difficult selection of damaged growing follicles from ovaries recently treated with lower doses of CPA and a hampered ability to identify and discard those with reduced viability for the culture.

Keywords: female fertility preservation, cyclophosphamide, in vitro culture and maturation, prepubertal ovary, chemotherapy, ovarian follicle isolation

\section{INTRODUCTION}

Improvements in diagnostic methods allowing early cancer diagnosis and improvements in cancer treatment have both increased cancer patients' survival rate globally. However, cancer treatment, especially chemotherapy and radiotherapy, can cause premature ovarian failure and infertility (1). For a young cancer survivor, this is a hard pathway to face. Thus, efforts have been made to develop methods for fertility preservation, and current guidelines recommend timely discussions with young adult patients, children, and their families on feasible fertility preservative methods as early as possible before the treatment starts aiming at offering the full range of options $(2,3)$.

Although well-established female fertility preservative methods including the cryopreservation of mature oocytes or embryos are available worldwide for adult patients, these are not applicable to young girls. Ovarian tissue cryopreservation can be offered instead in these cases (4). The method does not require hormonal stimulation, and the tissue is usually retrieved using minimally invasive surgery, which has been reported by programs for fertility preservation (5). However, many young patients need to start a treatment without delay, and data are lacking regarding the usefulness of ovarian tissue retrieved after the chemotherapy rounds have been already initiated.

Up to date, the only currently developed method to regain fertility using the cryopreserved ovarian tissue is by retransplantation of the tissue to the patient. The ovarian follicles will then grow and develop to allow the performance of assisted reproductive treatments or even natural conceptions (6). A few hundreds of successful cases using cryopreserved ovarian tissue have been reported worldwide; however, a high number of women cannot undergo transplantation due to the risk of reseeding malignant cells back within the transplanted tissue. There is hence an urgent need for developing novel methods to use the ovarian tissue in the future, as the numbers of women undergoing these procedures are increasing (7).

In this study, we approached two challenging clinical situations using a translational research model. We wished to investigate the feasibility of follicle isolation from prepubertal mice ovaries recently treated with a gonadotoxic cytostatic drug, cyclophosphamide (CPA) (8), and the reproductive potential of these follicles regarding the final achievement of mature competent oocytes. Three different doses of CPA were tested

Abbreviations: CPA, cyclophosphamide; $\alpha$-MEM, $\alpha$-minimal essential medium; MII, metaphase II; AMH, anti-Müllerian hormone; IVM, in vitro maturation; PFD, primordial follicle depletion; HSCT, hematologic stem cells transplantation. vs. a control group without treatment. In all groups, isolated follicles were thereafter in vitro cultured and matured. The culture system allowed the evaluation of individual follicle growth, hormonal production, oocyte maturation, and spindle structure and chromosomal configurations in mature oocytes. To our knowledge, there is limited knowledge on the feasibility of performing fertility preservative procedures after gonadotoxic cancer treatment has been initiated, especially at prepubertal stage $(9,10)$.

\section{MATERIALS AND METHODS}

All chemicals used in this study were purchased from SigmaAldrich $^{\circledR}$ or Gibco, Thermo Fisher Scientific ${ }^{\circledR}$, unless otherwise indicated.

\section{Animals and Grouping}

Twenty in-house breeding 12-day-old B6CBA/F1 female mice were randomly assigned ( $\mathrm{n}=5$ /group) into three groups treated with different doses of CPA $(100,75$, or $50 \mathrm{mg} / \mathrm{kg})$ or into a control group without treatment. CPA was freshly prepared in a $0.9 \% \mathrm{NaCl}$ solution and intraperitoneally injected. All the mice were sacrificed 3 days after CPA injection, and the ovaries were collected into Leibovitz's 15 media supplemented with $10 \%$ fetal bovine serum, $100 \mathrm{IU} / \mathrm{ml}$ penicillin, and $100 \mu \mathrm{g} / \mathrm{ml}$ streptomycin, at $37^{\circ} \mathrm{C}$. The doses of $\mathrm{CPA}$ chosen for this study have been validated in previous experimental studies that have also considered their equivalence to human treatment $(11,12)$.

All animal procedures were approved by Karolinska Institutet and the regional ethics committee for animal research in accordance with the Animal Protection Law, the Animal Protection Regulation, the Regulation of the Swedish National Board for Laboratory Animals, identified Dnr 1372 (date: January 24, 2018). All procedures were conducted in accordance with accepted standards of humane animal care.

\section{Follicle Isolation, In Vitro Culture, and Maturation}

Follicle isolation was performed immediately after ovary collection. Under a stereomicroscope $\left(\right.$ Nikon $\left.^{\circledR}\right)$, the ovaries were cleaned up of the surrounding tissue, and follicles were isolated mechanically using micro-fine U-100 insulin syringes (0.3 ml, BD Medical). Secondary follicles with 100-130 $\mu \mathrm{m}$ diameter, two or more layers of granulosa cells, a round and central oocyte, and some theca cells attached were selected for culture. Ovarian follicle culture was performed as previously 
described $(10,13,14)$. Briefly, selected secondary follicles were individually cultured in drops of a droplet system $(20 \mu \mathrm{l}$ per drop, 10 drops per dish) containing culture medium. The culture medium was $\alpha$-minimal essential medium ( $\alpha$-MEM) GlutaMAX supplemented with $5 \%$ fetal bovine serum, $10 \mu \mathrm{g} / \mathrm{ml}$ transferrin, 5 $\mu \mathrm{g} / \mathrm{ml}$ insulin, and $100 \mathrm{mIU} / \mathrm{ml}$ recombinant follicle-stimulating hormone (GONAL-F). The droplet system was covered with mineral oil and kept in a humidified incubator at $37^{\circ} \mathrm{C}$ with $5 \%$ $\mathrm{CO}_{2}$. Isolation, selection, and start of culture day was designated as day 0 and the last day as day 12 . Every other day, the follicles were observed under an inverted microscope $(100 \times)\left(\right.$ Nikon $\left.^{\circledR}\right)$ to record morphological characteristics and follicular size using a calibrated ocular micrometer $\left(\right.$ Nikon $\left.^{\circledR}\right)$. Follicular size was estimated as the mean diameter obtained with two perpendicular measures including the granulosa cell mass without the theca cells. Culture medium was renewed every other day by collecting $10 \mu \mathrm{l}$ of culture medium and adding $10 \mu \mathrm{l}$ offresh culture medium. On culture days 4,8 , and 12 , the culture medium collected was diluted in $90 \mu \mathrm{l} \alpha$ MEM GlutaMAX with bovine serum albumin $(40 \mathrm{mg} / \mathrm{ml})$ and stored at $-20^{\circ} \mathrm{C}$ for further analysis.

On day 12 of culture, follicles that reached at least $200 \mu \mathrm{m}$ of diameter, presented a clear granulosa cell proliferation, and had a visible round oocyte were classified as growing follicles that survived throughout the in vitro culture. Additionally, follicles with at least $450 \mu \mathrm{m}$ diameter were selected for in vitro maturation. In vitro maturation was performed by transferring the selected follicles at day 12 to a microdrop system similar to the culture system, but in which $1.5 \mathrm{IU} / \mathrm{ml}$ recombinant human chorionic gonadotrophins and $5 \mathrm{ng} / \mathrm{ml}$ recombinant epidermal growth factor were added to the culture medium. Follicles were incubated in a humidified atmosphere at $37^{\circ} \mathrm{C}$ and $5 \% \mathrm{CO}_{2}$, and maturation status was verified $16-20 \mathrm{~h}$ later. After verifying the cumulus-oocyte complex formation, the oocytes of the follicles that formed cumulus-oocyte complex were denuded, and their maturation status were evaluated under an inverted microscope. Oocytes with a visible polar body were classified as mature oocytes (metaphase II, MII).

\section{Hormone Assays}

The culture medium collected on days 4,8 , and 12 were used to measure the secretion of $17 \beta$-estradiol and anti-Müllerian hormone $(\mathrm{AMH})$. Hormonal assays were performed using commercially available enzyme-linked immunoassay kits for 17 $\beta$-estradiol (ab108667, Abcam) and AMH (RK02588, ABclonal) following the manufacturers' protocols. The limits of sensitivity for $17 \beta$-estradiol and AMH were 8.68 and $53.3 \mathrm{pg} / \mathrm{ml}$, respectively. For each hormone, duplicate measurements were performed using the collected culture medium at days 4,8 , and 12 of culture. For each time, in each group, the culture media of five follicles with similar growth features and from which resulted mature oocytes were pooled together to reach the required volume sample amount for the assay.

\section{Spindle and Chromosome Analysis of In Vitro Matured Oocytes}

After in vitro maturation (IVM), denuded mature oocytes (MII) were selected and washed in a washing buffer (Dulbecco's phosphate-buffered saline) containing $0.1 \%$ polyvinyl alcohol. The oocytes were then fixed with $2 \%$ formaldehyde in washing buffer containing $0.2 \%$ Triton X-100 for $40 \mathrm{~min}$. After fixation, the oocytes were incubated overnight at $4^{\circ} \mathrm{C}$ in a blocking buffer (washing buffer supplemented with $1 \%$ bovine serum albumin). Then, the oocytes were incubated for $40 \mathrm{~min}$ in the blocking buffer supplemented with $10 \%$ fetal bovine serum. Antibodies used were diluted in the blocking buffer. The oocytes were incubated with mouse monoclonal anti- $\alpha$-tubulin antibody (T9026, 1:1,000) for $45 \mathrm{~min}$ followed by a 40-min incubation with Alexa Fluor 488-labeled goat antimouse IgG antibodies (ab150113, Abcam, 1:200) at $37^{\circ} \mathrm{C}$. Then, $10 \mu \mathrm{g} / \mathrm{ml}$ propidium iodide (81845) was added to the oocytes for 20-min incubation. Finally, oocytes were mounted between a coverslip and a microscope slide with Prolong Diamond Antifade mountant (P36965, Invitrogen). All the above-described procedures were performed under a stereomicroscope. The slides were kept at $4^{\circ} \mathrm{C}$ until confocal imaging.

Labeled tubulin and chromatin were assessed using a Nikon Eclipse $\mathrm{Ti}$ microscope equipped with the appropriate filter sets for analyzing Alexa Fluor 488 and propidium iodide with 100× oil immersion objective. Four to six MII oocyte images in each group were captured with an Andor iXon Ultra camera and analyzed using NIS Elements program. Image analyses were performed using Fiji software.

\section{Statistical Analysis}

Statistical analyses were performed using the GraphPad Prism 8.4.3 software package. Differences in follicular sizes, hormones levels on different culture days, and follicle yield per ovary between groups were tested by multiple t-tests. Comparisons of parameters other than follicle yield in Table 1 between CPA treated groups and control were performed by chi-square test (two-sided).

TABLE 1 | Follicle yields per ovary, in vitro growth characteristics, and oocyte maturation status achieved by CPA-treated groups in comparison with controls.

\begin{tabular}{|c|c|c|c|c|c|c|c|}
\hline & Ovaries & Cultured follicles & Follicle yields/ovary & Attached within first 2 days & Survival (Day 12) & Put into maturation & MIl oocytes \\
\hline & $\mathbf{N}$ & $\mathbf{N}$ & Mean \pm SD & \multicolumn{4}{|c|}{$N(\%=100 \% \star N / C u l t u r e d$ follicles) } \\
\hline Control & 9 & 134 & $14.9 \pm 4.4$ & 113 (84.3\%) & 119 (88.8\%) & 107 (79.8\%) & 69 (51.5\%) \\
\hline 50 mg/kg & 9 & 170 & $18.9 \pm 6.4$ & $145(85.3 \%)$ & $133(78.2 \%)^{\mathrm{a}}$ & $115(67.6 \%)^{\mathrm{a}}$ & $56(32.9 \%)^{b}$ \\
\hline $75 \mathrm{mg} / \mathrm{kg}$ & 9 & 211 & $23.6 \pm 5.5^{\mathrm{b}}$ & $178(84.4 \%)$ & $172(81.5 \%)$ & $148(70.1 \%)^{\mathrm{a}}$ & $61(28.9 \%)^{\mathrm{C}}$ \\
\hline 100 mg/kg & 9 & 144 & $16.0 \pm 5.1$ & 130 (90.3\%) & 129 (89.6\%) & 110 (76.4\%) & 60 (41.7\%) \\
\hline
\end{tabular}

${ }^{a} p<0.05 ;{ }^{b} p<0.01 ;{ }^{c} p<0.001$. 


\section{RESULTS}

\section{Secondary Follicle Isolation Yield, In Vitro Growth, and Maturation}

A total of nine ovaries per group were used in this study. The yield of secondary follicles isolated per ovary did not differ significantly between 50 or $100 \mathrm{mg} / \mathrm{kg}$ CPA groups vs. control; however, in the $75 \mathrm{mg} / \mathrm{kg}$ group, the yield was significantly higher vs. control $(p<0.01$, Table 1).

The in vitro growth behavior of follicles during culture was similar among all groups regarding percentages of follicles attached to the culture dish during the first 2 days (Table 1) and growth curve features (Figure 1). Overall, the mean follicular size steadily increased throughout the culture but with different paces at different culture periods, with slower growth during the first half of culture and faster during the second half (Figure 1). The initial mean follicle diameter was $116.2 \mu \mathrm{m}$ among all the groups (Table 2). By the end of culture, follicles in all groups reached mean sizes ranging from 631.8 to 655.9 $\mu \mathrm{m}$ (Table 2) without significant differences. After 12 days in culture, nearly $90 \%$ of the follicles from the control and the $100 \mathrm{mg} / \mathrm{kg}$ groups survived. However, a lower follicle survival was observed in the 50 $\mathrm{mg} / \mathrm{kg}$ CPA group (78.2\%) vs. control (88.8\%), $p<0.05$ (Table 1). By the end of culture, significantly lower percentages of follicles reached the criteria to put into maturation in the 50 or $75 \mathrm{mg} / \mathrm{kg}$ CPA groups vs. control, whereas similar percentages were observed in the $100 \mathrm{mg} /$ $\mathrm{kg}$ and control groups.

After IVM, more mature oocytes were obtained in the control group (51.5\%) compared with the 50 and $75 \mathrm{mg} / \mathrm{kg}$ groups,

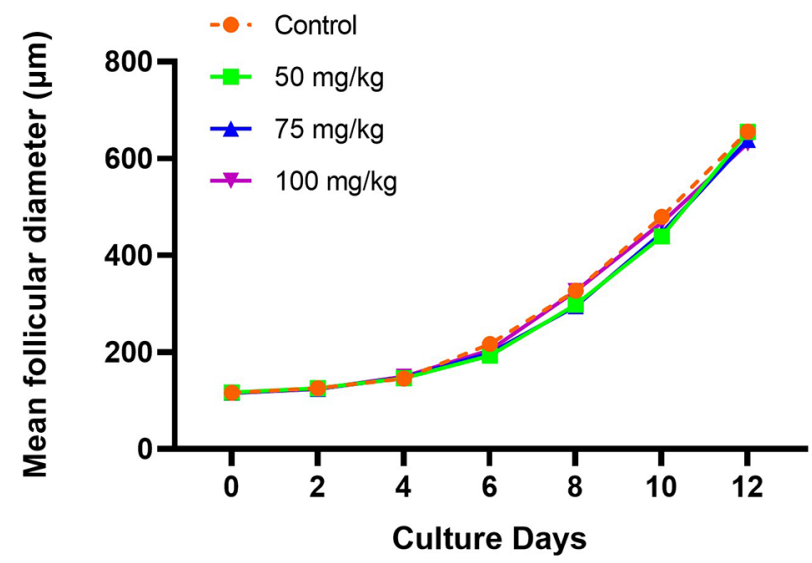

FIGURE 1 | Follicular growth curve in each group during the culture $(X$, culture days; $Y$, mean follicular diameter, $\mu \mathrm{m})$. where $32.9 \%$ and $28.9 \%$ were matured, respectively (Table 1). In the group treated with $100 \mathrm{mg} / \mathrm{kg} \mathrm{CPA}$, a similar percentage of mature oocytes was obtained compared to the control.

\section{Hormone Assays}

Follicle secretion of $17 \beta$-estradiol determined in culture media was similar among all groups, increasing from day 4 to 12 (Figure 2A). On the last day of culture (day 12), the levels of $17 \beta$-estradiol detected were similar among controls and the 75 and $100 \mathrm{mg} / \mathrm{kg}$ CPA groups. However, a lower secretion of $17 \beta$-estradiol was detected in the $50 \mathrm{mg} / \mathrm{kg}$ CPA group, compared to control $(11,023.57 \mathrm{pg} / \mathrm{ml}$ vs. $12,087.9 \mathrm{pg} / \mathrm{ml}, p<0.005)$.

Initial $\mathrm{AMH}$ secretion measured in culture medium on day 4 showed similar levels between control and $50 \mathrm{mg} / \mathrm{kg}$ CPA groups, whereas a significantly higher initial AMH secretion was found in the 75 and $100 \mathrm{mg} / \mathrm{kg} \mathrm{CPA}$ groups vs. control (Figure 2B). Over time, the AMH secretion gradually declined in the 50 and $75 \mathrm{mg} / \mathrm{kg}$ CPA groups and control, and the levels at day 12 did not differ among those groups vs control. However, the $100 \mathrm{mg} / \mathrm{kg}$ CPA group maintained a steady pace throughout the culture, and on day 12, the AMH level was 3.7 times of control $(p<0.0001)$.

\section{Spindle and Chromosome Configurations in Mature Oocytes}

In a normal MII oocyte, the spindle is bipolar barrel shaped, and the chromosomes are well-aligned on the metaphase equator in the center of the spindle. Within the MII oocytes observed, there was a trend that the spindle and chromosome structures in 100 $\mathrm{mg} / \mathrm{kg}$ CPA group were more similar to control, whereas spindle and chromosome abnormalities were frequently observed in 75 and $50 \mathrm{mg} / \mathrm{kg}$ CPA groups. As shown in Figure 3, mature oocytes in the control group displayed organized chromosomes at the center of the bipolar barrel-shaped spindle. Whereas in the 75 and $50 \mathrm{mg} / \mathrm{kg}$ CPA groups, chromosome misalignment and spindle defects were observed in mature oocytes. Chromosomes were, even though at the center of the spindle, not well-aligned on the metaphase equator; meanwhile fragmentary spindles were also observed. In mature oocytes obtained from the $100 \mathrm{mg} / \mathrm{kg}$ CPA group, the spindle was bipolar barrel shaped, and the chromosomes were well-aligned on the metaphase equator.

\section{DISCUSSION}

This study was designed to investigate the feasibility of obtaining mature oocytes after in vitro culture of follicles isolated from prepubertal mice recently treated with CPA. The follicle culture

TABLE 2 | Dynamic follicular sizes in each group during the culture (mean follicle diameter \pm standard deviation, $\mu$ m).

\begin{tabular}{|c|c|c|c|c|c|c|c|}
\hline & Do & D2 & D4 & D6 & D8 & D10 & D12 \\
\hline Control & $116.0 \pm 9.3$ & $125.5 \pm 14.6$ & $145.8 \pm 27.3$ & $217.3 \pm 102.8$ & $326.8 \pm 166.8$ & $479.6 \pm 191.9$ & $655.9 \pm 190.0$ \\
\hline $50 \mathrm{mg} / \mathrm{kg}$ & $116.9 \pm 8.6$ & $125.5 \pm 10.8$ & $146.6 \pm 27.3$ & $192.7 \pm 83.9$ & $298.1 \pm 157.6$ & $439.1 \pm 209.6$ & $655.0 \pm 199.3$ \\
\hline $75 \mathrm{mg} / \mathrm{kg}$ & $116.4 \pm 8.3$ & $124.4 \pm 12.4$ & $147.5 \pm 27.1$ & $198.4 \pm 73.1$ & $294.7 \pm 144.6$ & $446.2 \pm 187.0$ & $638.0 \pm 193.5$ \\
\hline $100 \mathrm{mg} / \mathrm{kg}$ & $115.6 \pm 8.5$ & $124.5 \pm 12.8$ & $149.5 \pm 32.0$ & $204.3 \pm 92.5$ & $326.3 \pm 169.9$ & $467.6 \pm 192.2$ & $631.8 \pm 179.3$ \\
\hline
\end{tabular}



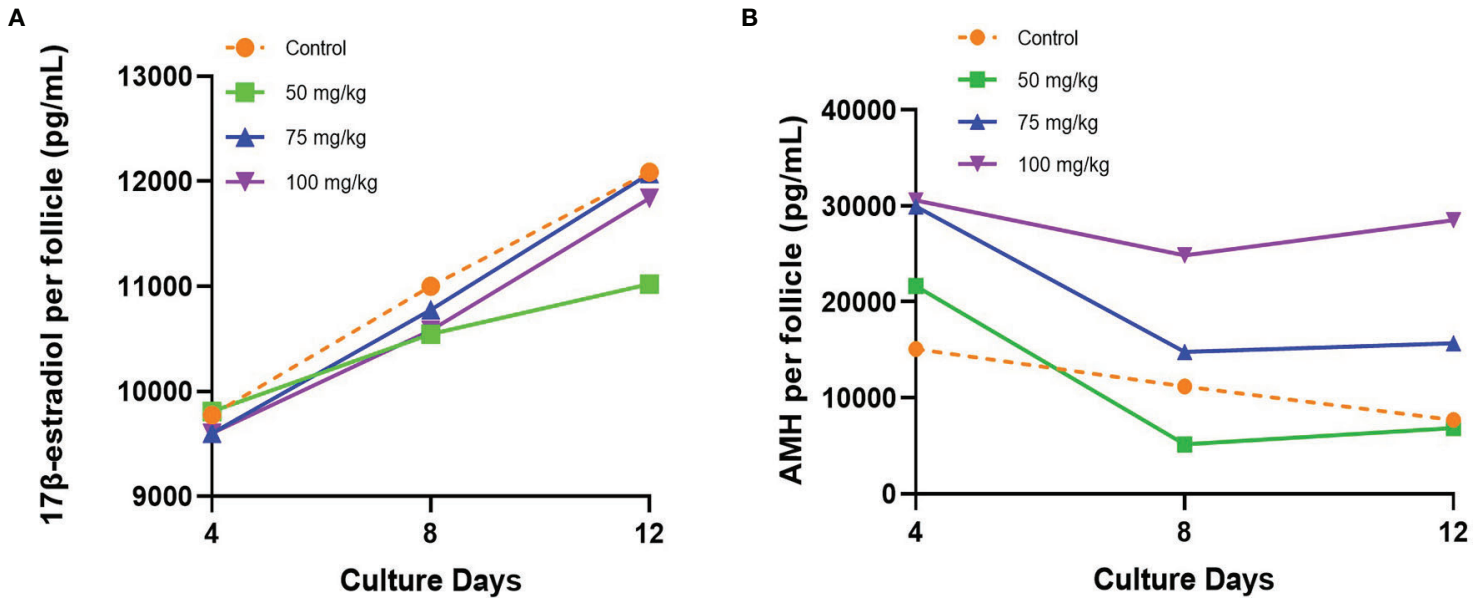

FIGURE 2 | (A) 17ß-estradiol and (B) AMH secreted by individual follicle into the culture medium during the culture.

and oocyte maturation methods have been previously validated in mice, and pups have been obtained using those methods (13-15). Spindle and chromosome arrangements were analyzed in this study to evaluate the quality of the mature oocytes obtained.

The ovary is a complex organ, where multiple regulations among activated follicles determine the ultimate fate of each

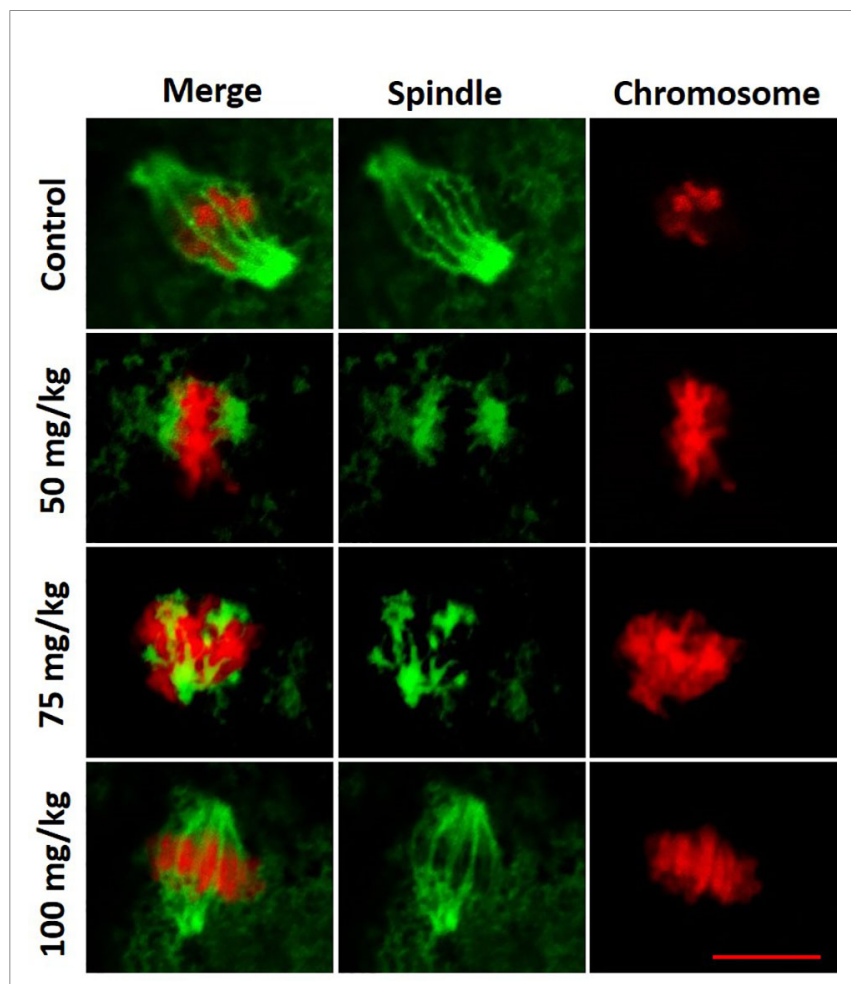

FIGURE 3 | Representative figures of spindle and chromosome configurations in MII oocytes from each group. Spindle fibers were detected by immunofluorescence for $\alpha$-tubulin (green), while DNA was stained with propidium iodide (red). Scale bar, $10 \mu \mathrm{m}$. individual follicle, whether it will proceed towards further growth or become atretic, resulting in the elimination of more than $90 \%$ of the activated follicles (16). Meanwhile, there is a critical surveillance system in the ovary, to remove dead follicles and follicles with damage (17-19). In our study, ovaries post CPA treatment may have had a proportion of secondary follicles physiologically impaired, and some were probably already targeted by the surveillance system due to CPA-induced damage. In our experimental conditions, we removed the secondary follicles from the ovarian environment to culture them individually, allowing them to escape the physiological inhibition and the surveillance system with the final aim to grow till mature oocytes.

Our findings showed that a large proportion of secondary follicles isolated from the ovaries of prepubertal mice after recent CPA treatment were not seriously affected by the CPA treatment. Those follicles could be supported to grow, and had spindle and chromosome configurations that were normal in the mature oocytes obtained after IVM, supporting the feasibility of our proposed method. Meanwhile, we found an interesting phenomenon towards the relationship between the efficiency of this method and the doses of CPA used, and we speculate this is somehow related to the mechanisms of how CPA induces primordial follicle depletion (PFD) in the ovaries.

According to the follicle selection criteria used in this culture system $(13,14)$, the yield of follicles/ovary obtained in the $75 \mathrm{mg} /$ $\mathrm{kg}$ group was significantly higher and in the $50 \mathrm{mg} / \mathrm{kg}$ group slightly higher than that of control, whereas follicle yield was smaller in the $100 \mathrm{mg} / \mathrm{kg}$ group. This could support an early overactivation mechanism, which has been proposed to explain the final PFD induced by gonadotoxic chemotherapy (20-23), or it could indicate that in the two lower-dose groups of this study, the CPA dose was not high enough to immediately damage the follicles or trigger apoptosis in them. In 50 and $75 \mathrm{mg} / \mathrm{kg}$ groups increased activation might have allowed to pick more secondary follicles for culture; however, at $100 \mathrm{mg} / \mathrm{kg}$, the follicle yield could have been additionally affected because the activated 
follicles were more rapidly damaged due to a high CPA dose, and many of them did not met the criteria to be cultured.

The gonadal toxicity of CPA is dose dependent, as previously demonstrated $(24,25)$. In our study, the observed similar culture outcomes between $100 \mathrm{mg} / \mathrm{kg}$ CPA and the control groups, while poorer outcomes in 50 and $75 \mathrm{mg} / \mathrm{kg}$ CPA groups, were surprising. These might indicate that the initial selection of potentially viable secondary follicles was easier in the $100 \mathrm{mg} / \mathrm{kg}$ group, due to more serious recent CPA treatment induced damage causing morphologically recognizable changes. The visible changes allowed to discard follicles with damage and to select follicles that had survived the damage and probably were qualified to overcome the surveillance system, while this selection was more difficult in the 50 and $75 \mathrm{mg} / \mathrm{kg}$ groups. Thus, a higher proportion of secondary follicles with minor but not morphologically detectable damage was selected for culture in the 50 and $75 \mathrm{mg} / \mathrm{kg}$ groups. However, during culture, it was evident that the competency of these follicles was impaired, and a lower survival rate was observed after 12 days of in vitro culture. Additionally, lower percentages of cultured follicles from these groups reached the criteria for IVM, lower percentages of MII oocytes were obtained, and abnormal spindle and damaged chromosome structures were more frequently observed in the oocytes of these two groups.

The observed higher levels of AMH in 75 and $100 \mathrm{mg} / \mathrm{kg} \mathrm{CPA}$ groups on day 4, and continuously higher in $100 \mathrm{mg} / \mathrm{kg}$ CPA group till day 12, might also support plausible primordial follicle activation after CPA in vivo treatment (20-23). This is not contradictory to the observation of reduced serum AMH levels by CPA treatment in studies in vivo $(26,27)$. Overactivation in vivo can initially lead to follicles sense through molecular communications and induce enhanced secretion of $\mathrm{AMH}$ to inhibit further overactivation through a paracrine regulation (28-30). Thereafter, the activated follicles may suffer direct damage by CPA or by lacking of growth support; thus, the AMH secretion becomes reduced in the long run. In this study, $72 \mathrm{~h}$ after CPA treatment, the activated follicles were isolated and individually cultured in vitro, leaving them free from subsequent CPA damage, but the initial effect that enhanced $\mathrm{AMH}$ production seemed to be kept, and the length of duration keeping this feature seemed to be dose dependent. As an alternative explanation, AMH could promote the growth of preantral follicles during in vitro culture through an autocrine effect, as supported by some studies (31-33). In our study, follicles isolated from $100 \mathrm{mg} / \mathrm{kg}$ CPA-treated mice ovaries might had been real survivors, secreting more AMH to promote their own growths. More investigations will be needed to further explain this phenomenon.

Since it is known that CPA is genotoxic (34), thus it is important to investigate the normality of the mature oocytes obtained after recent CPA treatment to guarantee the safety of our method. In addition to spindle and chromosome structure analysis, more investigations should be performed in the future, such as chromosome aberration tests and the final fertilization ability of the oocytes to evaluate normality of embryo development. On the other hand, clinical data from young female patients that have undergone ovarian tissue cryopreservation after several chemotherapy rounds and even after hematologic stem cells transplantation (HSCT) has demonstrated presence of primordial ovarian follicles in the cryopreserved tissue when the ovarian tissue was retrieved during childhood or adolescence (35). Moreover, ovarian tissue retrieved following HSCT conditioning at pubertal age has demonstrated full functionality after retransplantation, allowing two normal pregnancies, as recently reported (36).

Our experimental model deserves further translational investigation using human ovarian tissue. Methods for isolation and culture of human ovarian follicles obtaining mature oocytes have been reported (37). Further development of the methods hereby described is needed for the establishment of fertility preservative methods that can be performed after initiation of gonadotoxic chemotherapeutic treatment and that overcome the need of ovarian tissue retransplantation.

\section{DATA AVAILABILITY STATEMENT}

The raw data supporting the conclusions of this article will be made available by the authors, without undue reservation.

\section{ETHICS STATEMENT}

The animal study was reviewed and approved by Karolinska Institutet and the regional ethics committee for animal research.

\section{AUTHOR CONTRIBUTIONS}

$\mathrm{XH}, \mathrm{AA}$, and KR-W designed the experimental setting for the study. $\mathrm{XH}, \mathrm{AA}, \mathrm{LV}-\mathrm{R}, \mathrm{ASL}, \mathrm{CD}$ and SAdM performed the experiments. $\mathrm{XH}$ had the main responsibility for data analysis and wrote the first manuscript draft. All authors critically revised the manuscript. K-RW provided administrative support and funding. All authors contributed to the article and approved the submitted version.

\section{FUNDING}

This study was funded by the Swedish Childhood Cancer Foundation (PR2016-0115, PR2020-0136), the Swedish Cancer Society (CAN 2017/704, 200170 F), the Swedish Research Council (Dnr 2020-02230), Radiumhemmets Research Funds Grant for clinical researchers 2020-2025, the Stockholm County Council (FoUI-953912), and the Karolinska Institutet Research grants in pediatrics from the Birgitta and Carl-Axel Rydbeck Donation, 2020-00339 to KR-W. Doctoral candidate XH has been supported by the China Scholarship Council.

\section{ACKNOWLEDGMENTS}

We sincerely thank Raoul Kuiper at the FENO Core Facility at Karolinska Institutet, Stockholm, Sweden, for expert technical assistance, and Professor Jonas Bergh and Susanne Agartz, for administrative support and for providing an excellent experimental platform. 


\section{REFERENCES}

1. Grady MC. Preconception and the Young Cancer Survivor. Maternal Child Health J (2006) 10(5 Suppl):S165-8. doi: 10.1007/s10995-006-0103-1

2. Oktay K, Harvey BE, Partridge AH, Quinn GP, Reinecke J, Taylor HS, et al. Fertility Preservation in Patients With Cancer: ASCO Clinical Practice Guideline Update. J Clin Oncol (2018) 36(19):1994-2001. doi: 10.1200/JCO.2018.78.1914

3. ESHRE Guideline Group on Female Fertility Preservation, Anderson RA, Amant F, Braat D, D'Angelo A, Chuva de Sousa Lopes SM, et al. ESHRE Guideline: Female Fertility Preservation. Hum Reprod Open (2020) 2020(4): hoaa052. doi: 10.1093/hropen/hoaa052

4. Rodriguez-Wallberg KA, Oktay K. Fertility Preservation Medicine: Options for Young Adults and Children With Cancer. J Pediatr Hematol Oncol (2010) 32(5):390-6. doi: 10.1097/MPH.0b013e3181dce339

5. Rodriguez-Wallberg KA, Marklund A, Lundberg F, Wikander I, Milenkovic M, Anastacio A, et al. A Prospective Study of Women and Girls Undergoing Fertility Preservation Due to Oncologic and non-Oncologic Indications in Sweden-Trends in Patients' Choices and Benefit of the Chosen Methods After Long-Term Follow Up. Acta Obstet Gynecol Scand (2019) 98(5):604-15. doi: 10.1111/aogs.13559

6. Rodriguez-Wallberg KA, Oktay K. Recent Advances in Oocyte and Ovarian Tissue Cryopreservation and Transplantation. Best Pract Res Clin Obstet Gynaecol (2012) 26(3):391-405. doi: 10.1016/j.bpobgyn.2012.01.001

7. Rodriguez-Wallberg KA, Tanbo T, Tinkanen H, Thurin-Kjellberg A, Nedstrand E, Kitlinski ML, et al. Ovarian Tissue Cryopreservation and Transplantation Among Alternatives for Fertility Preservation in the Nordic Countries Compilation of 20 Years of Multicenter Experience. Acta Obstetricia Gynecol Scandinavica (2016) 95(9):1015-26. doi: 10.1111/aogs.12934

8. Meirow D, Biederman H, Anderson RA, Wallace WH. Toxicity of Chemotherapy and Radiation on Female Reproduction. Clin Obstet Gynecol (2010) 53(4):727-39. doi: 10.1097/GRF.0b013e3181f96b54

9. Asadi Azarbaijani B, Sheikhi M, Oskam IC, Nurmio M, Laine T, Tinkanen H, et al. Effect of Previous Chemotherapy on the Quality of Cryopreserved Human Ovarian Tissue In Vitro. PloS One (2015) 10(7):e0133985. doi: 10.1371/journal.pone.0133985

10. Anastácio A, Waterstone M, Hao X, Poirot C, Rodriguez-Wallberg KA. Ovarian Follicles Rescued 3 Days After Cyclophosphamide Treatment in Adolescent Mice: An Experimental Study Aiming at Maximizing Methods for Fertility Preservation Through In Vitro Follicle Culture. Int J Mol Sci (2019) 20(24):6190. doi: 10.3390/ijms20246190

11. Yang W, Ma Y, Jin J, Ren P, Zhou H, Xu S, et al. Cyclophosphamide Exposure Causes Long-Term Detrimental Effect of Oocytes Developmental Competence Through Affecting the Epigenetic Modification and Maternal Factors' Transcription During Oocyte Growth. Front Cell Dev Biol (2021) 9 (1444):682060. doi: 10.3389/fcell.2021.682060

12. Meirow D, Lewis H, Nugent D, Epstein M. Subclinical Depletion of Primordial Follicular Reserve in Mice Treated With Cyclophosphamide: Clinical Importance and Proposed Accurate Investigative Tool. Hum Reprod (1999) 14(7):1903-7. doi: 10.1093/humrep/14.7.1903

13. Cortvrindt R, Smitz J, Van Steirteghem AC. In Vitro Maturation, Fertilization and Embryo Development of Immature Oocytes From Early Preantral Follicles From Prepuberal Mice in a Simplified Culture System. Hum Reprod (1996) 11(12):2656-66. doi: 10.1093/oxfordjournals.humrep.a019188

14. Anastacio A, Rodriguez-Wallberg KA, Chardonnet S, Pionneau C, Fédérici C, Almeida Santos T, et al. Protein Profile of Mouse Ovarian Follicles Grown In Vitro. Mol Hum Reprod (2017) 23(12):827-41. doi: 10.1093/molehr/gax056

15. Morohaku K, Tanimoto R, Sasaki K, Kawahara-Miki R, Kono T, Hayashi K, et al. Complete In Vitro Generation of Fertile Oocytes From Mouse Primordial Germ Cells. Proc Natl Acad Sci USA (2016) 113(32):9021-6. doi: $10.1073 /$ pnas. 1603817113

16. Gougeon A. Dynamics of Follicular Growth in the Human: A Model From Preliminary Results. Hum Reprod (1986) 1(2):7. doi: 10.1093/ oxfordjournals.humrep.a136365

17. Ashwood-Smith MJ, Edwards RG. DNA Repair by Oocytes. Mol Hum Reprod (1996) 2(1):46-51. doi: 10.1093/molehr/2.1.46

18. Tilly JL. Commuting the Death Sentence: How Oocytes Strive to Survive. Nat Rev Mol Cell Biol (2001) 2(11):838-48. doi: 10.1038/35099086
19. Winship AL, Stringer JM, Liew SH, Hutt KJ. The Importance of DNA Repair for Maintaining Oocyte Quality in Response to Anti-Cancer Treatments, Environmental Toxins and Maternal Ageing. Hum Reprod Update (2018) 24 (2):119-34. doi: 10.1093/humupd/dmy002

20. Chang EM, Lim E, Yoon S, Jeong K, Bae S, Lee DR, et al. Cisplatin Induces Overactivation of the Dormant Primordial Follicle Through PTEN/AKT/ FOXO3a Pathway Which Leads to Loss of Ovarian Reserve in Mice. PloS One (2015) 10(12):e0144245. doi: 10.1371/journal.pone.0144245

21. Kalich-Philosoph L, Roness H, Carmely A, Fishel-Bartal M, Ligumsky H, Paglin S, et al. Cyclophosphamide Triggers Follicle Activation and "Burnout"; AS101 Prevents Follicle Loss and Preserves Fertility. Sci Transl Med (2013) 5 (185):185ra62. doi: 10.1126/scitranslmed.3005402

22. Lande Y, Fisch B, Tsur A, Farhi J, Prag-Rosenberg R, Ben-Haroush A, et al. Short-Term Exposure of Human Ovarian Follicles to Cyclophosphamide Metabolites Seems to Promote Follicular Activation In Vitro. Reprod BioMed Online (2017) 34(1):104-14. doi: 10.1016/j.rbmo.2016.10.005

23. Zhou L, Xie Y, Li S, Liang Y, Qiu Q, Lin H, et al. Rapamycin Prevents Cyclophosphamide-Induced Over-Activation of Primordial Follicle Pool Through PI3K/Akt/mTOR Signaling Pathway In Vivo. J Ovarian Res (2017) 10(1):56. doi: 10.1186/s13048-017-0350-3

24. Mark-Kappeler CJ, Hoyer PB, Devine PJ. Xenobiotic Effects on Ovarian Preantral Follicles. Biol Reprod (2011) 85(5):871-83. doi: 10.1095/ biolreprod.111.091173

25. Khedr NF, Khedr NF. Protective Effect of Mirtazapine and Hesperidin on Cyclophosphamide-Induced Oxidative Damage and Infertility in Rat Ovaries. Exp Biol Med (Maywood NJ) (2015) 240(12):1682-9. doi: 10.1177/ 1535370215576304

26. Salian SR, Uppangala S, Cheredath A, D'Souza F, Kalthur G, Nayak VC, et al. Early Prepubertal Cyclophosphamide Exposure in Mice Results in Long-Term Loss of Ovarian Reserve, and Impaired Embryonic Development and Blastocyst Quality. PloS One (2020) 15(6):e0235140-e0235140. doi: 10.1371/ journal.pone.0235140

27. Yoo M, Tanaka T, Konishi H, Tanabe A, Taniguchi K, Komura K, et al. The Protective Effect of Testosterone on the Ovarian Reserve During Cyclophosphamide Treatment. Oncol Targets Ther (2020) 13:2987-95. doi: 10.2147/OTT.S242703

28. Visser JA, Themmen APN. Role of Anti-Müllerian Hormone and Bone Morphogenetic Proteins in the Regulation of FSH Sensitivity. Mol Cell Endocrinol (2014) 382(1):460-5. doi: 10.1016/j.mce.2013.08.012

29. Nilsson EE, Schindler R, Savenkova MI, Skinner MK. Inhibitory Actions of Anti-Mullerian Hormone (AMH) on Ovarian Primordial Follicle Assembly. PloS One (2011) 6(5):e20087. doi: 10.1371/journal.pone.0020087

30. Nilsson E, Rogers N, Sknner MK. Actions of Anti-Müllerian Hormone on the Ovarian Transcriptome to Inhibit Primordial to Primary Follicle Transition. Reproduction (2007) 134(2):209-21. doi: 10.1530/REP-07-0119

31. Xu J, Xu F, Lawson MS, Tkachenko OY, Ting AY, Kahl CA, et al. AntiMüllerian Hormone is a Survival Factor and Promotes the Growth of Rhesus Macaque Preantral Follicles During Matrix-Free Culture. Biol Reprod (2018) 98(2):197-207. doi: 10.1093/biolre/iox181

32. Baarends WM, Uilenbroek JT, Kramer P, Hoogerbrugge JW, van Leeuwen EC, Themmen AP, et al. Anti-Müllerian Hormone and Anti-Müllerian Hormone Type II Receptor Messenger Ribonucleic Acid Expression in Rat Ovaries During Postnatal Development, the Estrous Cycle, and Gonadotropin-Induced Follicle Growth. Endocrinology (1995) 136 (11):4951-62. doi: 10.1210/endo.136.11.7588229

33. Xu J, Xu F, Letaw JH, Park BS, Searles RP, Ferguson BM. Anti-Müllerian Hormone is Produced Heterogeneously in Primate Preantral Follicles and Is a Potential Biomarker for Follicle Growth and Oocyte Maturation In Vitro. J Assist Reprod Genet (2016) 33(12):1665-75. doi: 10.1007/s10815-016-0804-3

34. Kour J, Ali MN, Ganaie HA, Tabassum N. Amelioration of the Cyclophosphamide Induced Genotoxic Damage in Mice by the Ethanolic Extract of Equisetum Arvense. Toxicol Rep (2017) 4:226-33. doi: 10.1016/ j.toxrep.2017.05.001

35. Wikander I, Lundberg FE, Nilsson H, Borgström B, Rodriguez-Wallberg KA. A Prospective Study on Fertility Preservation in Prepubertal and Adolescent Girls Undergoing Hematological Stem Cell Transplantation. Front Oncol (2021) 11(2560):692834. doi: 10.3389/fonc.2021.692834 
36. Rodriguez-Wallberg KA, Milenkovic M, Papaikonomou K, Keros V, Gustafsson B, Sergouniotis F, et al. Successful Pregnancies After Transplantation of Ovarian Tissue Retrieved and Cryopreserved at Time of Childhood Acute Lymphoblastic Leukemia - A Case Report. Haematologica (2021) 106. doi: 10.3324/haematol.2021.278828

37. McLaughlin M, Albertini DF, Wallace WHB, Anderson RA, Telfer EE. Metaphase II Oocytes From Human Unilaminar Follicles Grown in a Multi-Step Culture System. Mol Hum Reprod (2018) 24(3):135-42. doi: 10.1093/molehr/gay002

Conflict of Interest: The authors declare that the research was conducted in the absence of any commercial or financial relationships that could be construed as a potential conflict of interest.
Publisher's Note: All claims expressed in this article are solely those of the authors and do not necessarily represent those of their affiliated organizations, or those of the publisher, the editors and the reviewers. Any product that may be evaluated in this article, or claim that may be made by its manufacturer, is not guaranteed or endorsed by the publisher.

Copyright (c) 2021 Hao, Anastácio, Viñals-Ribé, Santamaria Lacuesta, Diakaki, Alonso de Mena, Liu and Rodriguez-Wallberg. This is an open-access article distributed under the terms of the Creative Commons Attribution License (CC BY).

The use, distribution or reproduction in other forums is permitted, provided the original author(s) and the copyright owner(s) are credited and that the original publication in this journal is cited, in accordance with accepted academic practice. No use, distribution or reproduction is permitted which does not comply with these terms. 